\title{
ORTHOGONAL DIAGONAL LATIN SQUARES OF ORDER FOURTEEN
}

\author{
L. ZHU
}

(Received 28 July 1982)

Communicated by W. D. Wallis

\begin{abstract}
Orthogonal diagonal latin squares of order $n, O D L S(n)$, are orthogonal latin squares of order $n$ with transversals on both the main diagonal and the back diagonal of each square. It has been proven that $O D L S(n)$ exist for all $n$ except $n=2,3,6,10,14,15,18$ and 26, in which the first three are impossible. In this note an example of $O D L S(14)$ is given.
\end{abstract}

1980 Mathematics subject classification (Amer. Math. Soc.): 05 B 15.

Orthogonal diagonal latin squares of order $n, O D L S(n)$, are orthogonal latin squares of order $n$ with transversals on both the main diagonal and the back diagonal of each square. The following open question is asked by J. Denes and A. D. Keedwell [1]: Do there exist orthogonal diagonal latin squares of order $n$ distinct from the impossible orders 2, 3 and 6? Recently several authors [2], [3], [4], [5] have proved that $O D L S(n)$ exist for all $n$ except $n=2,3,6,10,14,15,18$ and 26 , in which the first three are impossible. In this note an example of $O D L S(14)$ is given.

First we construct the orthogonal latin squares of order 14 as follows. The first latin square $L_{1}=\left(a_{i j}\right)$ has

$$
\begin{gathered}
\left(a_{0,0}, a_{0,1}, \ldots, a_{0,9}\right)=(0,3, A, 4,7,9,5, C, B, D), \\
\left(a_{0,10}, a_{0,11}, a_{0,12}, a_{0,13}\right)=(1,8,2,6), \\
\left(a_{10,0}, a_{11,0}, a_{12,0}, a_{13,0}\right)=(6,8,5,7),
\end{gathered}
$$

The author would like to thank Professor W. D. Wallis for his help during the preparation of this note. (C) 1984 Australian Mathematical Society 0263-6115/84 \$A2.00 + 0.00 
and a latin square $Q_{1}$ of order 4 based on $\{A, B, C, D\}$ in its lower right corner. The other elements are determined by the following rules:

(i) when $0 \leqslant i, j \leqslant 9, a_{i j}=a_{i-1, j-1}+1$, where all calculations (including those performed on indices) are performed modulo 10 on the residues $0,1,2, \ldots, 9$, and $x+1=x$ for the "infinite" element $x \in\{A, B, C, D\}$;

(ii) when $1 \leqslant r \leqslant 9,10 \leqslant j \leqslant 13, a_{r, j}=a_{0, j}+r$, the calculations being reduced by modulo 10 ;

(iii) when $1 \leqslant r \leqslant 9,10 \leqslant i \leqslant 13, a_{i, r}=a_{i, 0}+r$, the calculations being reduced by modulo 10 .

The second latin square $L_{2}=\left(b_{i j}\right)$ has

$$
\begin{gathered}
\left(b_{0,0}, b_{0,1}, \ldots, b_{0,9}\right)=(0, C, 9, D, A, 4, B, 1,3,2), \\
\left(b_{0,10}, b_{0,11}, b_{0,12}, b_{0,13}\right)=(5,6,8,7), \\
\left(b_{10,0}, b_{11,0}, b_{12,0}, b_{13,0}\right)=(8,1,2,6),
\end{gathered}
$$

and a latin square $Q_{2}$ orthogonal to $Q_{1}$ in its lower right corner. The other elements are determined by the same rules. It is easy to see that $L_{1}$ and $L_{2}$ are orthogonal latin squares of order 14. If we take $Q_{1}$ and $Q_{2}$ to be orthogonal diagonal latin squares of order 4 , we obtain the squares $L_{1}$ and $L_{2}$ shown in Table 1 .

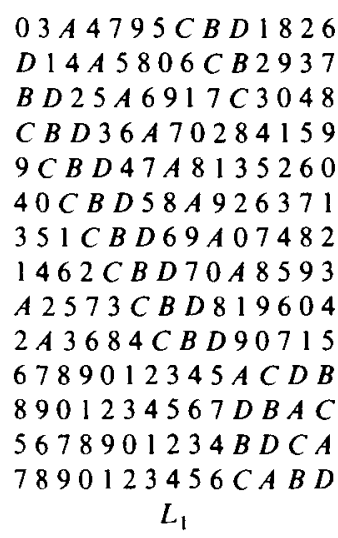

$03 A 4795 C B D 1826$ $D 14 A 5806 C B 2937$

3048

59 07482 $1462 C B D 70 A 8593$ $A 2573 C B D 819604$ $2 A 3684 C B D 90715$ $6789012345 A C D B$ $8901234567 D B A C$ $5678901234 B D C A$ $L_{\mathrm{l}}$

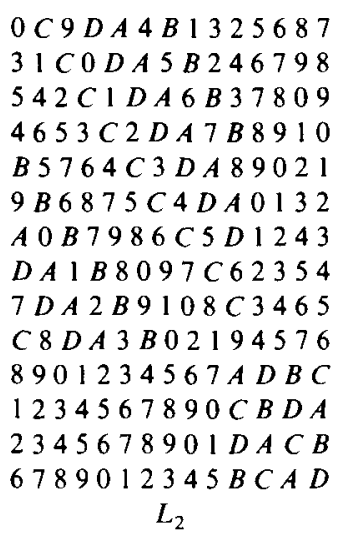

TABLE 1

It is seen that these squares have a common transversal down the diagonal. Another common transversal is cells $(0,5),(1,6),(2,7),(3,8)$ and $(4,9)$ and their transposes, together with the back diagonal of the subsquares $Q_{1}$ and $Q_{2}$. If rows and columns are permuted simultaneously, the diagonal is preserved, and it is possible to move the other transversal onto the back diagonal. Thus we get an example of orthogonal diagonal latin squares of order 14, illustrated in Table 2. 
$03 A 471826 D B C 59$
$D 14 A 52937 B C 608$
$B D 25 A 3048 C 7196$
$C B D 3641598207 A$
$9 C B D 45260318 A 7$
$67890 A C D B 54321$
$89012 D B A C 76543$
$56789 B D C A 43210$
$78901 C A B D 65432$
$2 A 36807159 D B C 4$
$A 2573960418 D B C$
$1462 C 8593 A 07 D B$
$351 C B 74820 A 96 D$
$40 C B D 637129 A 85$
$0 C 9 D A 5687231 B 4$
$31 C 0 D 679842 B 5 A$
$542 C 178093 B 6 A D$
$4653 C 8910 B 7 A D 2$
$B 576490218 A D 3 C$
$89012 A D B C 76543$
$12345 C B D A 09876$
$23456 D A C B 10987$
$67890 B C A D 54321$
$C 8 D A 345769120 B$
$7 D A 2 B 3465 C 8019$
$D A 1 B 823546 C 790$
$A 0 B 791243 D 5 C 68$
$9 B 6870132 A D 4 C 5$

TABLE 2

\section{References}

[1] J. Denes and A. D. Keedwell, Latin squares and their applications (Academic Press, New York, 1974).

[2] K. Heinrich and A. J. W. Hilton, 'Doubly diagonal orthogonal latin squares', to appear.

[3] W. D. Wallis and L. Zhu, 'Existence of orthogonal diagonal latin squares', Ars Combinatoria 12 (1981), 51-68.

[4] W. D. Wallis and L. Zhu, 'Some new orthogonal diagonal latin squares', J. Austral. Math. Soc. Ser. $A$, to appear.

[5] W. D. Wallis and L. Zhu, 'Four pairwise orthogonal diagonal latin squares of order 12', Utilitas Math. 21 C (1982), 205-207.

\section{Department of Mathematics \\ Kiangsu Teachers College \\ Soochow \\ China}

